\title{
Experimental observation of chiral phonons in monolayer WSe2
}

Hanyu Zhu, Jun Yi, Ming-Yang Li, Jun Xiao, Lifa Zhang, et al.

Hanyu Zhu, Jun Yi, Ming-Yang Li, Jun Xiao, Lifa Zhang, Chih-Wen Yang, Sui Yang, Robert A. Kaindl, Lain-Jong Li, Yuan Wang, Xiang Zhang,

"Experimental observation of chiral phonons in monolayer WSe2," Proc. SPIE 10916, Ultrafast Phenomena and Nanophotonics XXIII, 109160C (27

February 2019); doi: 10.1117/12.2510760

SPIE. Event: SPIE OPTO, 2019, San Francisco, California, United States 


\title{
Experimental Observation of Chiral Phonons in Monolayer WSe Hanyu Zhu ${ }^{\mathrm{a}, \mathrm{b}}$, Jun Yi ${ }^{\mathrm{a}}$, Ming-Yang Lic ${ }^{\mathrm{c}}$, Jun Xiao ${ }^{\mathrm{a}}$, Lifa Zhang ${ }^{\mathrm{d}}$, Chih-Wen Yang ${ }^{\mathrm{c}}$, Sui Yang ${ }^{\mathrm{a}}$, Robert A. Kaindl ${ }^{\mathrm{e}}$, Lain-Jong $\mathrm{Li}^{\mathrm{c}}$, Y Yan Wang ${ }^{\mathrm{a}, \mathrm{e}}$ \& Xiang Zhang ${ }^{\mathrm{a}}$, e, $\mathrm{f}$ \\ ${ }^{a}$ Nanoscale Science and Engineering Center, University of California, Berkeley, CA 94720, USA; ${ }^{b}$ Department of Materials Science and NanoEngineering, Rice University, Houston, TX 77005, USA; ${ }^{\mathrm{c}}$ Physical Sciences and Engineering Division, King Abdullah University of Science and Technology, Thuwal, 23955-6900, Kingdom of Saudi Arabia; ${ }^{\mathrm{d} D e p a r t m e n t ~ o f ~ P h y s i c s, ~ N a n j i n g ~}$ Normal University, Nanjing, Jiangsu 210023, China; ${ }^{\mathrm{C}}$ Materials Sciences Division, Lawrence

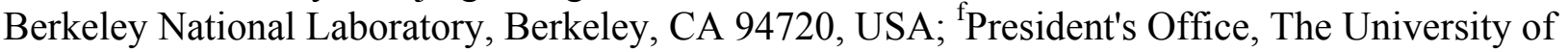 Hong Kong, Hong Kong, China
}

\begin{abstract}
Chirality characterizes an object that is not identical to its mirror image. In condensed matter physics, Fermions have been demonstrated to obtain chirality through structural and time-reversal symmetry breaking. These systems display unconventional electronic transport phenomena such as the quantum Hall effect and Weyl semimetals. However, for bosonic collective excitations in atomic lattices, chirality was only theoretically predicted and has never been observed. We experimentally show that phonons can exhibit intrinsic chirality in monolayer tungsten diselenide, whose lattice breaks the inversion symmetry and enables inequivalent electronic $\mathrm{K}$ and $-\mathrm{K}$ valley states. The time-reversal symmetry is also broken when we selectively excite the valley polarized holes by circularly polarized light. Brillouin-zone-boundary phonons are then optically created by the indirect infrared absorption through the hole-phonon interactions. The unidirectional intervalley transfer of holes ensures that only the phonon modes in one valley are excited. We found that such photons are chiral through the transient infrared circular dichroism, which proves the valley phonons responsible to the indirect absorption has non-zero pseudo-angular momentum. From the spectrum we further deduce the energy transferred to the phonons that agrees with both the first principle calculation and the double-resonance Raman spectroscopy. The chiral phonons have significant implications for electron-phonon coupling in solids, lattice-driven topological states, and energy efficient information processing.
\end{abstract}

Keywords: Chirality, phonon, valley, transition metal dichalcogenide, time-reversal symmetry breaking, indirect absorption, zone-boundary, intervalence band transition.

\section{INTRODUCTION}

Chirality reveals the symmetry breaking of a physical framework, its elementary excitation, and/or the interaction between these excitations. For example, chiral Fermions with spin-momentum locking can emerge in recently observed Weyl semimetals with inversion-symmetry-breaking lattices ${ }^{1}$. Electron chirality in graphene defined through pseudospin was found to cause unique transport properties like unconventional Landau quantization and Klein tunneling ${ }^{2,3}$. An optical helicity for excitons also emerges in monolayer transition metal dichalcogenides with broken inversion symmetry, leading to the experimental realization of valleytronics ${ }^{4}$. The outstanding question that has yet been experimentally investigated is whether bosonic collective excitations such as phonons can display chirality. Recently, the intrinsic chirality of phonons without applied external magnetic fields in an atomic lattice was predicted theoretically at the corners of the Brillouin zone of an asymmetric two-dimensional hexagonal lattice ${ }^{5}$. Because of the three-fold rotational symmetry of the crystal and the momentum vectors, the vibrational plane wave is comprised of unidirectional atomic circular rotation. Since the phonons in the same valley have distinct energy levels, the rotation is not the superposition of linear modes, in contrast to the circular polarization in non-chiral media ${ }^{6}$. The hypothetical chiral phonons are potentially important information carriers like the valley electrons ${ }^{7,8}$. But compared with electrons, they are charge-neutral and have much smaller electrical and magnetic dipole moment, and are thus potentially more robust against decoherence from electromagnetic interaction and noise- for the control of intervalley scattering ${ }^{9,10}$. In addition 
to functionalities on their own, the chiral phonons may find potential application in lattice-modulation-driven electronic phase transitions ${ }^{11,12}$, topological states ${ }^{13}$, and polaronic superconductivity ${ }^{14}$.

The first distinctive feature of chiral phonon is its non-zero pseudo-angular momentum (PAM) ${ }^{5}$. A particle's angular momentum is calculated by the phase change under a rotational transformation. This definition can be extended for quasi-particles in solid lattices with discrete spatial symmetry, as long as the rotation is in the crystalline symmetry group $^{15}$. For inversion-symmetry-breaking $2 \mathrm{D}$ hexagonal lattice like monolayer $\mathrm{WSe}_{2}$, phonons with well-defined PAM are located at $\Gamma, \mathrm{K}$ and $-\mathrm{K}$ points in the reciprocal space (Video. 1(a)) $)^{5}$. Because they inherit the three-fold rotational $\left(\mathrm{C}_{3}\right)$ symmetry of the lattice, their function of motion must be $\mathrm{C}_{3}$ invariant except for a phase difference, i.e. circular. The $\mathrm{K}$ and $-\mathrm{K}$ phonons are particularly interesting because there is no reflection line that preserves both the lattice and their momentum. Therefore, the atomic rotation cannot be reversed without changing its momentum or energy. For example, if we attempt to reverse the rotational direction of the Se atoms in longitudinal optical phonon $\mathrm{LO}(\mathrm{K})$ while keeping the relative phase determined by the $\boldsymbol{q}=\boldsymbol{K}$ momentum, the mode changes to a longitudinal acoustic phonon LA(K) that oscillates at a different frequency (Video 1(b) and 1(c); the timing is not proportional to the actual period, so the frequency difference is not shown in the video). It means that unlike the $\Gamma$ phonon, such a rotation cannot be decomposed into orthogonal linear vibrations with $\pm \pi / 2$ phase shift. On the other hand, in order to maintain the same mode, the relative phase must be reversed, resulting in a phonon at the $-\mathrm{K}$ point.

(a)

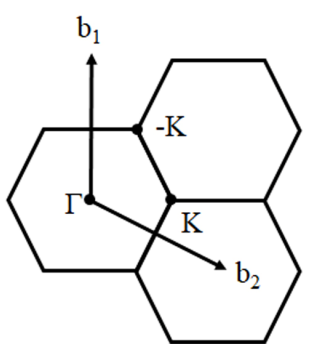

(b)

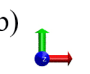

$\mathbb{t} \Leftrightarrow$

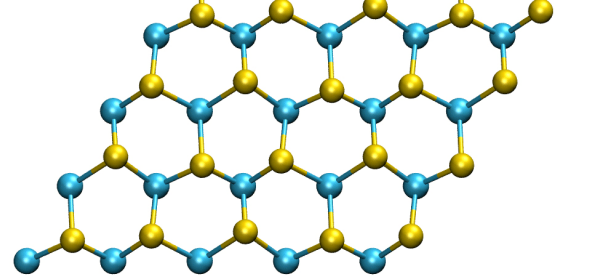

(c)

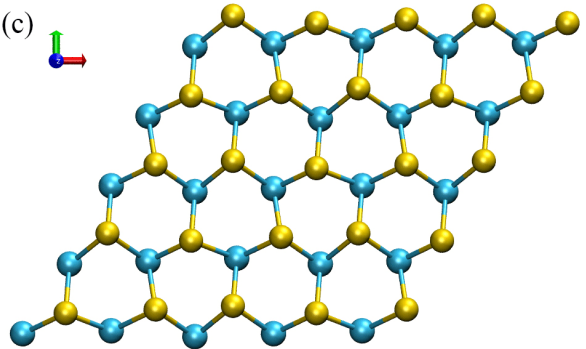

Figure 1. "Video1.mp4" The symmetry origin of chiral phonons. (a) In the reciprocal space of a hexagonal lattice, there are three high-symmetry points $\Gamma, \mathrm{K}$ and $-\mathrm{K}$ that preserves the lattice's three-fold rotational symmetry. The phonon modes with these momenta have well-defined pseudo-angular momentum. (b) The atomic motion of $\mathrm{LO}(\mathrm{K})$ and (c) LA(K) modes in monolayer $\mathrm{WSe}_{2}$ (Video 1). The blue and yellow balls represent the $\mathrm{W}$ and Se atoms, respectively. With the same momentum, the rotation of Se is reversed at the cost of bringing W into motion. http://dx.doi.org/10.1117/12.2510760.1

(a)

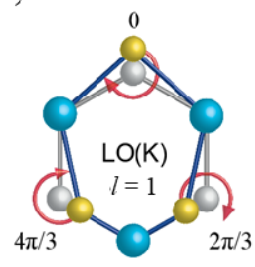

(b)

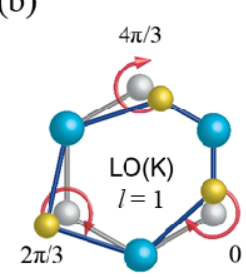

Figure 2. The PAM is obtained by the phase gain after the $\mathrm{C}_{3}$ rotation. Because of the 3 -fold symmetry, the PAM has three choices: 0 and \pm 1 . Rotating the atomic configuration of the $\operatorname{LO}(\mathrm{K})$ phonon in (a) by $120^{\circ}$ to (b) results in a $-2 \pi / 3$ phase change, indicating the PAM is 1 for this mode.

We deduce the phonon PAM $l$ for each mode from the phase change after counter-clockwise $120^{\circ}$ rotation: $\hat{\mathrm{C}}_{3}\left(u_{\mathrm{k}}\right)=$ $e^{-i(2 \pi / 3) l} u_{\mathrm{k}}$, where $u_{\mathrm{k}}$ is the function of atomic motion (Fig. 2). Since $\mathrm{LA}(\mathrm{K})$ is always $\mathrm{C}_{3}$ symmetric, it has $l=0$, while $\mathrm{LO}(\mathrm{K})$ gains negative phase after rotation (becomes ahead of time) and thus $l=1$. The $\mathrm{K}$ phonons with different PAM are completely non-degenerate due to the mass difference between $\mathrm{W}$ and Se. The lifting of degeneracy guarantees that each mode has a distinctive selection rule for the electron-phonon and optical scattering. These features differentiate the intrinsically chiral phonons from any previously investigated phonons where circular atomic motion results from superposing linear polarized eigenmodes ${ }^{6,16,17}$. The intrinsic chirality in the absence of an external magnetic flux is also distinguished from magnetic-field-induced splitting of originally degenerate phonon modes ${ }^{18,19}$. Finally, both the physical origin and properties of the atomic chiral phonons are fundamentally different from previous phononic-crystalbased chirality with broken time-reversal symmetry, topological edge states, or geometric modulation ${ }^{20-25}$. 


\section{EXPERIMENTAL METHODS}

The deterministic creation of chiral phonon is characterized by the optical pump-probe spectroscopy (Fig. 3). First, a hole at the valence band edge at the K valley is injected by the left-circular-polarized (LCP) optical pump pulse ${ }^{26,27}$. The $\mathrm{K}$-valley-polarized hole relaxes to the valence band edge with initial linear momentum $p_{\mathrm{i}}=-\boldsymbol{K}$. It can transit to the $-\mathrm{K}$ point $\left(p_{\mathrm{f}}=\boldsymbol{K}\right)$ by emitting a $\mathrm{K}$ phonon $\left(q=p_{\mathrm{i}}-p_{\mathrm{f}}=-2 \boldsymbol{K}=\boldsymbol{K}\right)$, but the energy of the new state does not match any real band at the $-\mathrm{K}$ valley due to the large spin-orbit coupling. Then we send an infrared pulse to satisfy the energy conservation and place the hole in the spin-split band at $-\mathrm{K}$. Since the hole states have zero PAM, the PAM of the phonon must be equal to the spin angular momentum of the absorbed infrared photon. Therefore, the LCP pulse controls the creation of only $\mathrm{LO}(\mathrm{K})$ phonons. Eventually, we recognize the final spin-split state in the opposite valley by the energy and the right-circular polarization (RCP) of its radiative decay. Since this is a four-particle scattering process, the linear momentum, PAM and energy of the phonon modes can be determined by characterizing all the other particles involved, i.e. the initial hole, the infrared photon and the final hole.

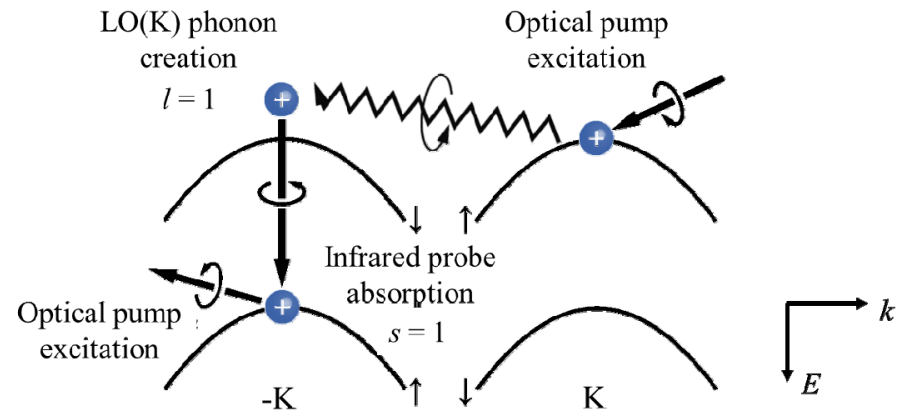

Figure 3. The valence band diagram of the transient indirect absorption spectroscopy. Deeper hole levels indicate higher carrier energy. The experiment was conducted using a tunable Ti:Sapphire pulsed laser at $80 \mathrm{MHz}$ repetition rate. An optical parametric oscillator converts the pulse to one signal beam, frequency-doubled to $1.82-1.97 \mathrm{eV}$ as the pump to excite holes, and one idler beam $0.42-0.51 \mathrm{eV}$ as the probe to supplement the energy difference due to the spin-orbit splitting. The sample was held in a transmission cryostat at $82 \mathrm{~K}$. The fluorescence was collected with a photomultiplier collecting in the range of $1.98-2.23 \mathrm{eV}$.

We only consider the phonon creation process and neglect the phonon annihilation process because of the cryogenic temperature of the sample, which results in very low phonon number for all $\mathrm{K}$ and $-\mathrm{K}$ modes. The pump beam size is spread to about $70 \mu \mathrm{m}$ so as to keep a maximum pump pulse of $15 \mathrm{uJ} / \mathrm{cm}^{-2}$ and peak optical doping density of about $3 \times 10^{12} \mathrm{~cm}^{-2}$. The density is small enough to avoid band renormalization ${ }^{28}$, and the Fermi level of trions are only a few $\mathrm{meV}$ above the band edge. Given the heat capacitance of $\mathrm{WSe}_{2}$ at $82 \mathrm{~K}$ is $6.7 \times 10^{-8} \mathrm{~J} / \mathrm{cm}^{-2} \cdot \mathrm{K}^{29}$, the lattice temperature rise following the pump is around $10 \mathrm{~K}$, which is still well below the Debye temperature of the phonon mode at $\mathrm{K} / \mathrm{K}$ with the lowest energy $\left(T_{\theta}(\mathrm{TA}(\mathrm{K}))=134 \mathrm{~K}\right)$. Besides, the selection should neither be affected by a small momentum distribution of the initial and final holes due to the finite thermal energy about a few percent of the reciprocal vector. This is because like the optical valley-selectivity, the contrast of electron-phonon coupling strength remains large in the vicinity of $\mathrm{K}$ and $-\mathrm{K}$ even if the PAM is only defined on these points.

\section{RESULTS AND DISCUSSION}

\subsection{Determination of the initial hole states}

Under circularly-polarized pump excitation, the majority of holes maintain their linear momentum near the original valley as shown by the helicity of the photoluminescence (PL, Fig. 4(a)). Because of the natural doping of the samples, the PL is dominated by trion emission centered around $1.703 \pm 0.006 \mathrm{eV}, 33 \mathrm{meV}$ below the A-exciton absorption peak. We find that the PL emission is much broader than reported exfoliated crystal because of inhomogeneous broadening. Therefore, we estimate the uncertainty of emission energy by multiple measurements. The PL helicity is not necessarily equal to the actual valley polarization at different time after the pulse excitation since the luminescence is integrated for all time. Nevertheless, it provides a qualitative indicator for the degree of initial momentum selection. Due to the significant spin-orbit splitting in the conduction band of $\mathrm{WSe}_{2}$, the initial state with the lowest energy that contains an 
excited hole is not the optically bright trion. Rather, most of the holes would form a dark trion $32 \pm 1 \mathrm{meV}$ below the bright trion (Fig. 4(b) and (c)) ${ }^{30}$. Thus, the dark trion energy is calculated to be $1.671 \pm 0.006 \mathrm{eV}$.

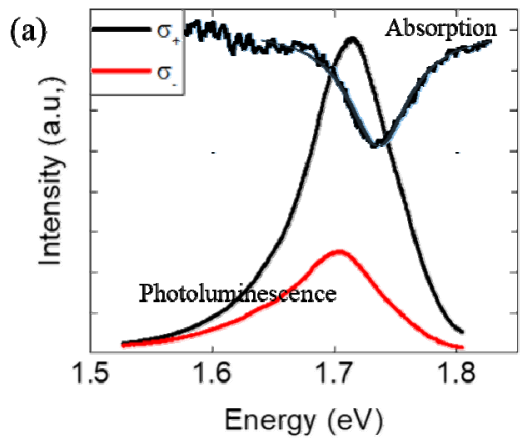

(b)

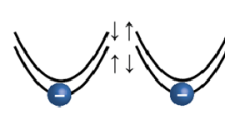

(c)
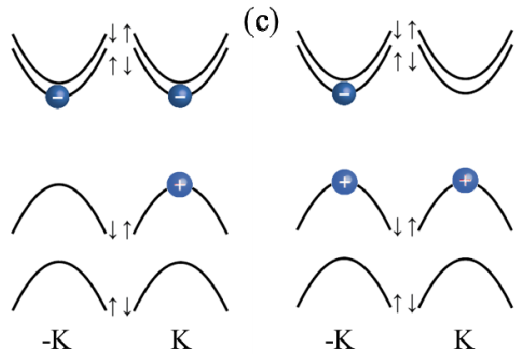

4. Characterization of the monolayer $\mathrm{WSe}_{2}$ sample. The PL clearly shows that the sample is doped, but either (b) a dark A-trion with an extra electron or (c) a dark A-trion with an extra hole could be the possible initial state of the majority of holes.

\subsection{Determination of the final hole states}

The final linear momentum of holes after the intervalence band transition (IVBT) ${ }^{31}$ is read out by the polarization of their luminescence. The polarized luminescence is defined as the difference of luminescence intensity with the same polarization as the pump light $\left(I_{\text {same }}\right)$ and the opposite polarization $\left(I_{\mathrm{oppo}}\right)$. We find $I_{\text {same }}-I_{\mathrm{oppo}}$ is a function of the probe delay time $\tau$ (Fig. 5(a)). The polarization is positive for $\tau<0.2 \mathrm{ps,} \mathrm{indicating} \mathrm{that} \mathrm{the} \mathrm{spin-flipping} \mathrm{intravalley} \mathrm{IVBT} \mathrm{has}$ a higher probability when the IR probe arrives immediately after the excitation of holes. This is because spin is not strictly conserved for optical transitions away from the high symmetry points (Fig. 5(b)). However, the process is forbidden at the valley center with incident light perpendicular to the lattice plane, so its magnitude decays with a lifetime of $0.2 \mathrm{ps}$ as the non-equilibrium carriers thermalize to the band edge ${ }^{32,33}$. After that, the luminescence switches polarization as a result of the dominant spin-conserving intervalley IVBT. It shows that the majority of final holes have a linear momentum opposite to the initial holes. Finally, the depolarization plus the decay of the hole population yields a joint lifetime of about 2 ps for the polarized luminescence, which agrees with previous exciton studies ${ }^{34}$.

(a)

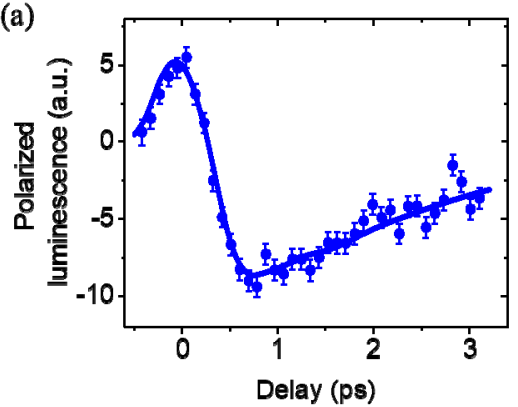

(b)

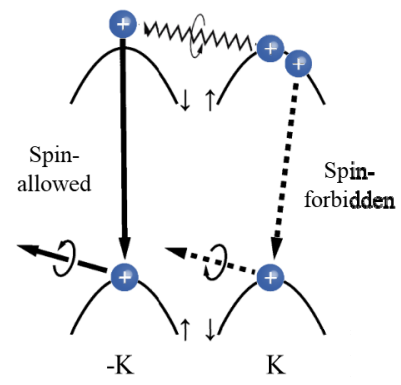

Figure 5. The dynamics of polarized luminescence. (a) The polarization of the B-exciton emission is initially aligned with the A-exciton excitation, indicating a spin-forbidden intravalley transition process for hot carriers in (b). Instead, the band edge holes are more likely to go through intervalley transition.

To obtain the energy of the final holes, we measure the spectrum of the polarized luminescence with $\tau=0.8$ ps (Fig. 6(a)). Each point in Fig. 6(a) is acquired by adding a narrow band pass filter to the detector. The signal intensity, about a few counts per second, is normalized by the integrated transmission spectrum of each filter, whose center of gravity gives the x-coordinate. The optically bright state is a B-trion as shown in Fig. 6(b) and (c), resulting from a simple move of holes from Fig. 4(b) and (c) to the spin-split band in the other valley by absorbing a photon and emit a phonon. To accurately measure the B-trion energy, we found a probe energy centered at $462 \mathrm{meV}$ gives large emission signal 
without bringing much extra energy to broaden the emission peak. Under this condition, the Lorentzian fit of the spectrum yields a final hole energy of $2.090 \pm 0.005 \mathrm{eV}$. The normalized helicity at $\tau=0.8 \mathrm{ps}$ is around $-5 \%$ to $-10 \%$. It is reasonable that the helicity of spin-split hole emission is lower than the band edge emission, because there is no spinorbit splitting to block the valley depolarization of holes with high energy even if the intervalley transition has $100 \%$ yield.

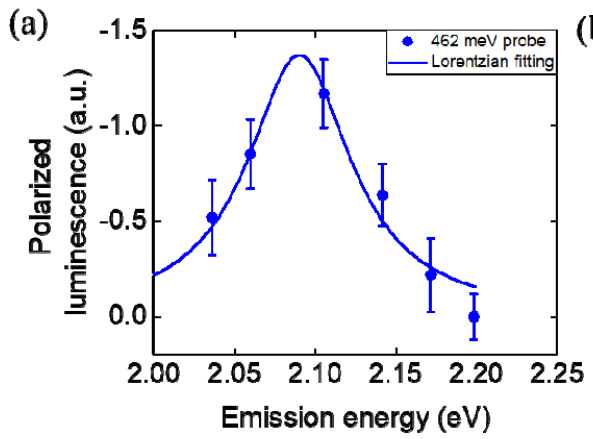

(b)
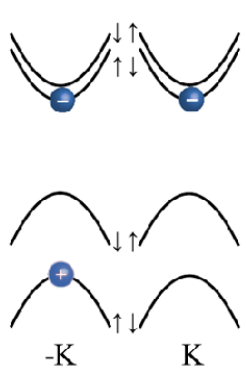

(c)<smiles>C=COCCOC</smiles><smiles>CCCOCC</smiles>

Figure 6. (a) The emission spectrum of the B-trions. (b) and (c) shows the possible B-trion configurations after the intervalley transition of holes corresponding to the dark A-trions in Fig. 4 (b) and (c).

\subsection{Measuring the angular momentum of the photon and the phonon}

The phonon-assisted IR absorption is expected to be circularly dichroic due to the chiral electron-phonon interaction. We measured the difference of IR absorption when the probe has the same polarization as the pump light $\left(\alpha_{\text {same }}\right)$, and that for the opposite polarization $\left(\alpha_{\mathrm{oppo}}\right)$. The observed infrared circular dichroism $\left(\mathrm{CD}=\alpha_{\text {same }}-\alpha_{\mathrm{oppo}}\right)$ for all delay time proved that the system indeed becomes chiral, indicating a non-zero PAM for the phonons (Fig. 7(a)). With LCP excitation, the intervalley transition of holes from the K valley can either absorb an LCP IR photon to produce LO phonons, or absorb an RCP IR photon to produce TA/A $A_{1}$ phonons. Our result shows that the scattering cross-sections of the LO process is much larger than that of the TA/A $/ \mathrm{A}_{1}$ process (Fig. 7(b)). Meanwhile, it is also possible for the angular momentum of the IR photon to be transferred to the electronic spin instead of the phonon. The initial intravalley IVBT gives positive CD because the electronic spin flips from $-1 / 2$ in the spin-split band to $+1 / 2$ at the valence band edge at the $\mathrm{K}$ point. However, this contribution decays fast as a function of IR delay with a life time of $0.2 \mathrm{ps}$, in accordance to that of Fig. 5(a). Thus, the intervalley phonon-creating transition is the dominant source of positive CD for $\tau \geq 0.8 \mathrm{ps}$.

(a)

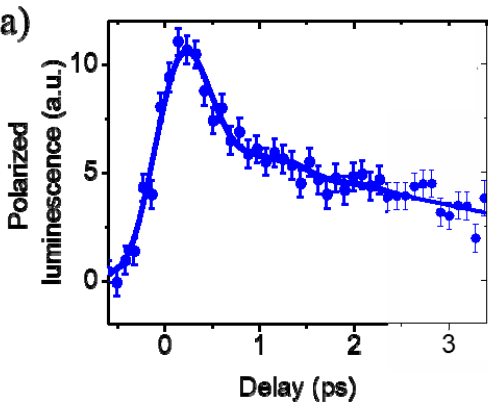

(b)

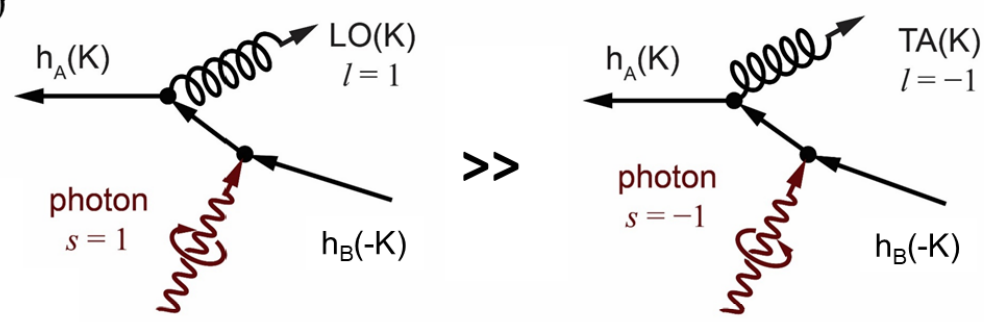

Figure 7. (a) Transient circular dichroism in the indirect absorption as a function of probe delay. The positive CD immediately after the pump reflects the angular momentum transfer to the spin. (b) The remnant positive CD after $0.8 \mathrm{ps}$ shows the difference of electron-phonon coupling strength for the left- and right-handed phonon modes.

The momentum must have been transferred to phonons instead of defects, as seen from the order-of-magnitude weaker defect-assisted one-phonon Raman scattering compared with the two-phonon process (Fig. 8) ${ }^{35,36}$. There are two types of second-order scattering processes, one involving one phonon and one defect, the other involving two phonons. Their relative intensity tells the strength of intervalley scattering by defects compared with by phonons. Here the two-phonon scattering near $400 \mathrm{~cm}^{-1}(2 * 24.8 \mathrm{meV})$ is order-of-magnitude stronger than the defect scattering near $200 \mathrm{~cm}^{-1}$.

Therefore, the intervalley scattering almost all comes from phonon. 
(a)

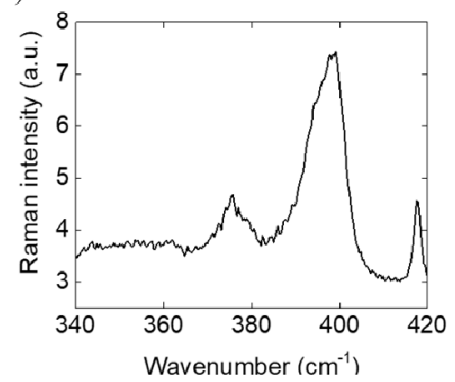

(b)

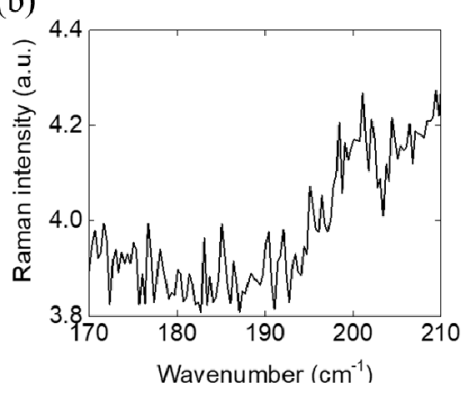

Figure 8: The second-order Raman scattering by (a) two-phonon and (b) one-phonon plus one photon process measured by $633 \mathrm{~nm}$ near-resonant excitation, indicating dominant phonon contribution to the intervalley scattering.

Another momentum transfer process, the directional intervalley transfer through electron-electron interactions ${ }^{37}$ is excluded because the pump is not in resonance with the A-exciton, and the signal is linearly proportional to the hole density (Fig. 9). The electron-electron interaction should be stronger at higher carrier density, and thus the signal is expected to be superlinearly dependent on power. Yet in our experiment, while the integrated high-energy emission between 2.05-2.19 eV contains strong contribution from two-photon absorption of the pump light as shown by the quadratic fitting, the IR-induced difference is still proportional to pump power. It suggests that our signal comes from a single-hole process, and successfully suppresses the two-photon luminescence background. The exchange interaction reduces the polarization of the holes but should not create an opposite polarization ${ }^{38}$.

(a)

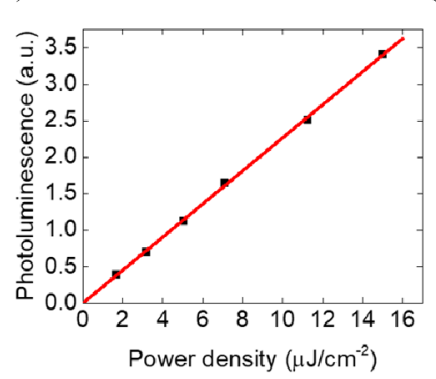

(b)

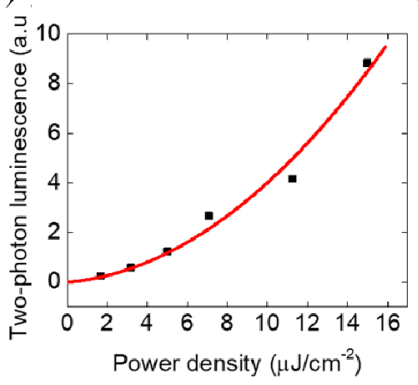

(c)

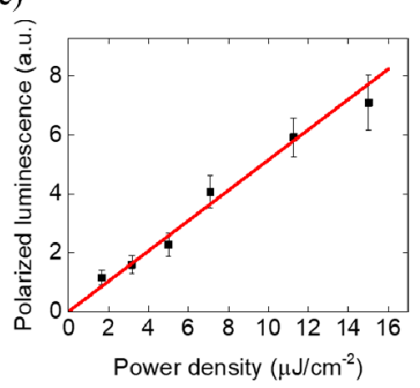

Figure 9: Dependence to pump power of (a) total photoluminescence between 1.5-1.9 eV, (b) total luminescence signal collected between 2.05-2.19 eV and (c) IR-induced polarized luminescence signal. The linearity of signal rules out the intervalley transfer by electron-electron interaction.

The absolute $\mathrm{CD}$ is estimated by the above analysis to be on the order of $10^{-8}$. This very small value is the reason why we use the luminescence to measure IR absorption instead of using standard transient reflectivity measurement. Besides the concern with signal to noise ratio, the reflectivity can be affected by many other effects than intervalence band transition, such as Kerr rotation, band renormalization, free-carrier absorption etc. By contrast, although the luminescence is weak, it provides clean signature of the intervalley transition to the spin-split holes.

\subsection{Measuring the energy of the photon and the phonon}

The energy of the most prominent phonon mode in the indirect absorption is measured according to energy conservation. The energy sum of the incoming particles, the infrared photon and the initial holes must be equal to that of the outgoing particles, the chiral phonon and the final holes. We observe that the CD spectrum at $\tau=0.8 \mathrm{ps}$ (Fig. 10(a)) is a step function with a clear threshold near $0.448 \pm 0.002 \mathrm{eV}$, broadened by the spectral width of the IR pulse. The shape corresponds to a transition from a hole at the valence edge to a band with parabolic dispersion in two dimensions (Fig. 10(b)). Therefore, the above threshold represents the photon energy required for creating a final hole with zero kinetic energy. We find that this value is distinguished from the intra-excitonic transition which has a much lower energy ${ }^{39,40}$, as well as from the exciton dissociation which needs higher energy ${ }^{41}$. Summarizing the previously measured energy of 
the initial and final hole states, we deduce the phonon energy $E_{\text {phonon }}=E_{\text {photon }}+E_{\mathrm{i}}-E_{\mathrm{f}}=29 \pm 8 \mathrm{meV}$. The value agrees with both the first-principles calculation (Fig. 10(c)) and the aforementioned two-phonon Raman spectrum (Fig. 8(a)). The uncertainty may be reduced in the future by improving the uniformity of the sample and the spectral resolution of the B-exciton emission. Better spectroscopy will also potentially reveal details about the chiral phonon-exciton coupling that are not resolvable with the current signal-to-noise ratio, such as the contribution from LA phonons and the various pathways of the indirect transition.

(a)

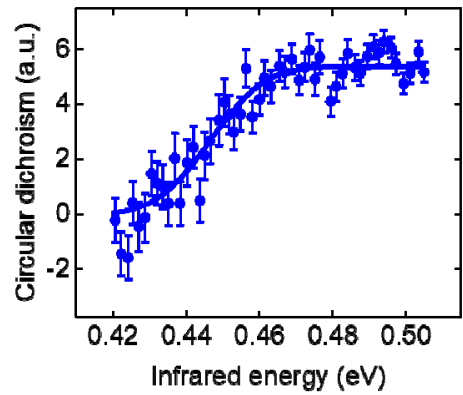

(b)

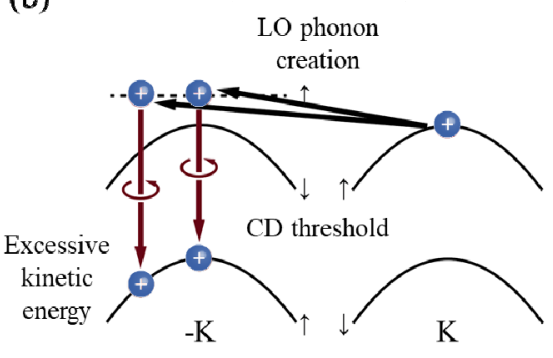

(c)

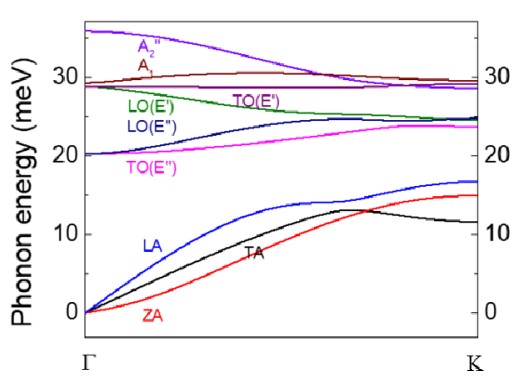

Figure 10: (a) The spectrum of the transient CD acquired at $\tau=0.8$ ps shows a photonic energy threshold of $0.448 \pm 0.002$ $\mathrm{eV}$. The error bars are standard error calculated from multiple measurements, and the threshold uncertainty is the standard error of the fitting parameter. (b) The step-shaped spectrum comes from the two-dimensional excitonic density of states. The phonon dispersion is flat compared with electrons, so the virtual band of phonon scattered holes is also flat. The IR absorption threshold corresponds to a transition at the -K point, while higher photon energy is needed for the transition away from the $-\mathrm{K}$ point. (c) The experimentally derived energy of $\mathrm{LO}(\mathrm{K})$ is $29 \pm 8 \mathrm{meV}$, agreeing with the phononic band structure from first principle calculations.

\section{CONCLUSION}

In conclusion, we have experimentally observed the chiral phonon with non-zero pseudo-angular momentum at $\mathrm{K}$ and $\mathrm{K}$ valleys in monolayer $\mathrm{WSe}_{2}$. These atomic rotational modes cannot be decomposed into a superposition of linear vibrations in the same valley. The phonon chirality originates from the unique combination of inversion symmetry breaking of the lattice and the three-fold rotational symmetry of the momentum vectors. We measured the circular dichroism of the phonon-assisted indirect transition as a consequence of the selection rule of hole-phonon interactions. Our findings of chiral phonons are fundamentally important for potential experimental tests of quantum theories with chiral bosons ${ }^{42,43}$ in the solid state. It also provides a possible route for controlling valley and spin through electronphonon scattering and strong spin-phonon interactions ${ }^{44,17}$. Furthermore, the lifting of degeneracy by chirality offers robust PAM information against decoherence and long-range perturbation, and offers a new degree of freedom to the design and implementation of phononic circuitry ${ }^{45,46}$ at the atomic scale without magnetic fields ${ }^{47-49}$.

\section{ACKNOWLEDGEMENTS}

This work was primarily supported by the U.S. Department of Energy, Office of Science, Basic Energy Sciences, Materials Sciences and Engineering Division under contract no. DE-AC02-05-CH11231 within the van der Waals Heterostructures program (KCWF16) for sample preparation and theory and data analysis, and within the Subwavelength Metamaterials Program (KC12XZ) for optical design and measurement. R. A. K. was supported by DOE BES, Materials Sciences and Engineering Division under contract no. DE-AC02-05-CH11231 within the Ultrafast Materials Science program (KC2203) for mid-IR frequency conversion. J.Y. acknowledges a scholarship from the China Scholarship Council (CSC) under grant no. 201606310094. L.Z. acknowledges support from the National Natural Science Foundation of China (grant no. 11574154). L.-J.L. acknowledges support from the King Abdullah University of Science and Technology through a Competitive Research Grant (CRG5) for monolayer $\mathrm{WSe}_{2}$ synthesis. All data needed to evaluate the conclusions in the paper are present in the paper and the supplementary materials. 


\section{REFERENCES}

[1] Hasan, M. Z., Xu, S.-Y., Belopolski, I. and Huang, S.-M., "Discovery of Weyl Fermion Semimetals and Topological Fermi Arc States,” Annu. Rev. Condens. Matter Phys. 8(1), 289-309 (2017).

[2] Haldane, F. D. M., "Model for a Quantum Hall Effect without Landau Levels: Condensed-Matter Realization of the 'Parity Anomaly,"” Phys. Rev. Lett. 61(18), 2015-2018 (1988).

[3] Das Sarma, S., Adam, S., Hwang, E. H. and Rossi, E., "Electronic transport in two-dimensional graphene," Rev. Mod. Phys. 83(2), 407-470 (2011).

[4] Schaibley, J. R., Yu, H., Clark, G., Rivera, P., Ross, J. S., Seyler, K. L., Yao, W. and Xu, X., "Valleytronics in 2D materials," Nat. Rev. Mater. 1(11), 16055 (2016).

[5] Zhang, L. and Niu, Q., "Chiral Phonons at High-Symmetry Points in Monolayer Hexagonal Lattices," Phys. Rev. Lett. 115(11), 115502 (2015).

[6] Katsuki, H., Delagnes, J. C., Hosaka, K., Ishioka, K., Chiba, H., Zijlstra, E. S., Garcia, M. E., Takahashi, H., Watanabe, K., Kitajima, M., Matsumoto, Y., Nakamura, K. G. and Ohmori, K., "All-optical control and visualization of ultrafast two-dimensional atomic motions in a single crystal of bismuth," Nat. Commun. 4, ncomms3801 (2013).

[7] Jones, A. M., Yu, H., Ghimire, N. J., Wu, S., Aivazian, G., Ross, J. S., Zhao, B., Yan, J., Mandrus, D. G., Xiao, D., Yao, W. and Xu, X., "Optical generation of excitonic valley coherence in monolayer WSe2," Nat. Nanotechnol. 8(9), 634-638 (2013).

[8] Hao, K., Moody, G., Wu, F., Dass, C. K., Xu, L., Chen, C.-H., Sun, L., Li, M.-Y., Li, L.-J., MacDonald, A. H. and Li, X., "Direct measurement of exciton valley coherence in monolayer WSe2," Nat. Phys. 12(7), 677-682 (2016).

[9] Zeng, H., Dai, J., Yao, W., Xiao, D. and Cui, X., "Valley polarization in MoS2 monolayers by optical pumping," Nat. Nanotechnol. 7(8), 490-493 (2012).

[10] Carvalho, B. R., Wang, Y., Mignuzzi, S., Roy, D., Terrones, M., Fantini, C., Crespi, V. H., Malard, L. M. and Pimenta, M. A., "Intervalley scattering by acoustic phonons in two-dimensional $\mathrm{MoS}_{2}$ revealed by doubleresonance Raman spectroscopy," Nat. Commun. 8, ncomms14670 (2017).

[11] Rini, M., Tobey, R., Dean, N., Itatani, J., Tomioka, Y., Tokura, Y., Schoenlein, R. W. and Cavalleri, A., "Control of the electronic phase of a manganite by mode-selective vibrational excitation," Nature 449(7158), 72 74 (2007).

[12] Först, M., Mankowsky, R. and Cavalleri, A., "Mode-Selective Control of the Crystal Lattice," Acc. Chem. Res. 48(2), 380-387 (2015).

[13] Jotzu, G., Messer, M., Desbuquois, R., Lebrat, M., Uehlinger, T., Greif, D. and Esslinger, T., "Experimental realization of the topological Haldane model with ultracold fermions," Nature 515(7526), 237-240 (2014).

[14] Kang, M., Jung, S. W., Shin, W. J., Sohn, Y., Ryu, S. H., Kim, T. K., Hoesch, M. and Kim, K. S., "Holstein polaron in a valley-degenerate two-dimensional semiconductor," Nat. Mater. 17(8), 676-680 (2018).

[15] Johnson, R. C., “Angular momentum on a lattice," Phys. Lett. B 114(2), 147-151 (1982).

[16] Chen, S.-Y., Zheng, C., Fuhrer, M. S. and Yan, J., "Helicity-Resolved Raman Scattering of MoS2, MoSe2, WS2, and WSe2 Atomic Layers," Nano Lett. 15(4), 2526-2532 (2015).

[17] Nova, T. F., Cartella, A., Cantaluppi, A., Först, M., Bossini, D., Mikhaylovskiy, R. V., Kimel, A. V., Merlin, R. and Cavalleri, A., "An effective magnetic field from optically driven phonons," Nat. Phys. 13(2), 132 (2017).

[18] Anastassakis, E., Burstein, E., Maradudin, A. A. and Minnick, R., "Morphic effects - III. Effects of an external magnetic field on the long wavelength optical phonons," J. Phys. Chem. Solids 33(2), 519-531 (1972).

[19] Schaack, G., "Observation of circularly polarized phonon states in an external magnetic field," J. Phys. C Solid State Phys. 9(11), L297 (1976).

[20] Spadoni, A., Ruzzene, M., Gonella, S. and Scarpa, F., "Phononic properties of hexagonal chiral lattices," Wave Motion 46(7), 435-450 (2009).

[21] Süsstrunk, R. and Huber, S. D., "Observation of phononic helical edge states in a mechanical topological insulator," Science 349(6243), 47-50 (2015).

[22] Mousavi, S. H., Khanikaev, A. B. and Wang, Z., "Topologically protected elastic waves in phononic metamaterials," Nat. Commun. 6, 8682 (2015).

[23] Wang, P., Lu, L. and Bertoldi, K., "Topological Phononic Crystals with One-Way Elastic Edge Waves," Phys. Rev. Lett. 115(10), 104302 (2015). 
[24] Lu, J., Qiu, C., Ke, M. and Liu, Z., "Valley Vortex States in Sonic Crystals," Phys. Rev. Lett. 116(9), 093901 (2016).

[25] Brendel, C., Peano, V., Painter, O. J. and Marquardt, F., "Pseudomagnetic fields for sound at the nanoscale," Proc. Natl. Acad. Sci. 114(17), E3390-E3395 (2017).

[26] Zhu, C. R., Zhang, K., Glazov, M., Urbaszek, B., Amand, T., Ji, Z. W., Liu, B. L. and Marie, X., "Exciton valley dynamics probed by Kerr rotation in WSe2 monolayers," Phys. Rev. B 90(16), 161302 (2014).

[27] Hsu, W.-T., Chen, Y.-L., Chen, C.-H., Liu, P.-S., Hou, T.-H., Li, L.-J. and Chang, W.-H., "Optically initialized robust valley-polarized holes in monolayer WSe2," Nat. Commun. 6, 8963 (2015).

[28] Chernikov, A., Ruppert, C., Hill, H. M., Rigosi, A. F. and Heinz, T. F., "Population inversion and giant bandgap renormalization in atomically thin WS2 layers," Nat. Photonics 9(7), 466-470 (2015).

[29] Bolgar, A. S., Trofimova, Z. A. and Yanaki, A. A., "Thermodynamic properties of tungsten diselenide in a broad temperature range," Sov. Powder Metall. Met. Ceram. 29(5), 382-385 (1990).

[30] Zhang, X.-X., Cao, T., Lu, Z., Lin, Y.-C., Zhang, F., Wang, Y., Li, Z., Hone, J. C., Robinson, J. A., Smirnov, D., Louie, S. G. and Heinz, T. F., "Magnetic brightening and control of dark excitons in monolayer WSe2," Nat. Nanotechnol. 12(9), 883-888 (2017).

[31] Braunstein, R., "Intervalence band transitions in gallium arsenide," J. Phys. Chem. Solids 8(Supplement C), 280-282 (1959).

[32] Ceballos, F., Cui, Q., Bellus, M. Z. and Zhao, H., "Exciton formation in monolayer transition metal dichalcogenides," Nanoscale 8(22), 11681-11688 (2016).

[33] Steinleitner, P., Merkl, P., Nagler, P., Mornhinweg, J., Schüller, C., Korn, T., Chernikov, A. and Huber, R., "Direct Observation of Ultrafast Exciton Formation in a Monolayer of WSe2," Nano Lett. 17(3), 1455-1460 (2017).

[34] Yan, T., Ye, J., Qiao, X., Tan, P. and Zhang, X., "Exciton valley dynamics in monolayer WSe2 probed by the two-color ultrafast Kerr rotation," Phys. Chem. Chem. Phys. 19(4), 3176-3181 (2017).

[35] Pimenta, M. A., Dresselhaus, G., Dresselhaus, M. S., Cançado, L. G., Jorio, A. and Saito, R., "Studying disorder in graphite-based systems by Raman spectroscopy," Phys. Chem. Chem. Phys. 9(11), 1276-1290 (2007).

[36] Mignuzzi, S., Pollard, A. J., Bonini, N., Brennan, B., Gilmore, I. S., Pimenta, M. A., Richards, D. and Roy, D., "Effect of disorder on Raman scattering of single-layer MoS2," Phys. Rev. B 91(19), 195411 (2015).

[37] Manca, M., Glazov, M. M., Robert, C., Cadiz, F., Taniguchi, T., Watanabe, K., Courtade, E., Amand, T., Renucci, P., Marie, X., Wang, G. and Urbaszek, B., "Enabling valley selective exciton scattering in monolayer $\mathrm{WSe}_{2}$ through upconversion," Nat. Commun. 8, ncomms14927 (2017).

[38] Yu, T. and Wu, M. W., "Valley depolarization due to intervalley and intravalley electron-hole exchange interactions in monolayer MoS2," Phys. Rev. B 89(20), 205303 (2014).

[39] Poellmann, C., Steinleitner, P., Leierseder, U., Nagler, P., Plechinger, G., Porer, M., Bratschitsch, R., Schüller, C., Korn, T. and Huber, R., "Resonant internal quantum transitions and femtosecond radiative decay of excitons in monolayer WSe2," Nat. Mater. 14(9), 889-893 (2015).

[40] Cha, S., Sung, J. H., Sim, S., Park, J., Heo, H., Jo, M.-H. and Choi, H., "1s-intraexcitonic dynamics in monolayer $\mathrm{MoS}_{2}$ probed by ultrafast mid-infrared spectroscopy,” Nat. Commun. 7, ncomms 10768 (2016).

[41] Ye, Z., Cao, T., O'Brien, K., Zhu, H., Yin, X., Wang, Y., Louie, S. G. and Zhang, X., "Probing excitonic dark states in single-layer tungsten disulphide," Nature 513(7517), 214-218 (2014).

[42] Sonnenschein, J., "Chiral bosons," Nucl. Phys. B 309(4), 752-770 (1988).

[43] Barreira, M. M. H. and Wotzasek, C., "Chiral-boson quantum mechanics," Phys. Rev. D 45(4), 1410-1415 (1992).

[44] Kiselev, A. A., "Circular phonons and optical magnetization of crystals," Phys. Status Solidi B 119(2), 525-530 (1983).

[45] Li, N., Ren, J., Wang, L., Zhang, G., Hänggi, P. and Li, B., "Colloquium: Phononics: Manipulating heat flow with electronic analogs and beyond," Rev. Mod. Phys. 84(3), 1045-1066 (2012).

[46] Liu, Y., Xu, Y., Zhang, S.-C. and Duan, W., "Model for topological phononics and phonon diode," Phys. Rev. B 96(6), $064106(2017)$.

[47] Strohm, C., Rikken, G. L. J. A. and Wyder, P., "Phenomenological Evidence for the Phonon Hall Effect," Phys. Rev. Lett. 95(15), 155901 (2005).

[48] Wang, Y.-T., Luan, P.-G. and Zhang, S., "Coriolis force induced topological order for classical mechanical vibrations," New J. Phys. 17(7), 073031 (2015).

[49] Liu, D. and Shi, J., "Circular Phonon Dichroism in Weyl Semimetals," Phys. Rev. Lett. 119(7), 075301 (2017). 